\title{
A low-swelling polymeric mixed conductor operating in aqueous electrolytes
}

Tommaso Nicolini, * Jokubas Surgailis, Achilleas Savva, Alberto D. Scaccabarozzi, Rana Nakar, Damien Thuau, Guillaume Wantz, Lee J. Richter, Olivier Dautel, Georges Hadziioannou, Natalie Stingelin*

Dr. T. Nicolini

Université de Bordeaux, CNRS Bordeaux INP/ENSCBP, Institut de Sciences Moléculaires UMR 5255, 16

Avenue Pey Berland, 33607 Pessac, France

tommaso.nicolini@enscbp.fr

J. Surgailis

King Abdullah University of Science and Technology, Organic Bioelectronics Lab., Thuwal 23955, Saudi

Arabia

Dr. Alberto D. Scaccabarozzi

King Abdullah University of Science and Technology, KAUST Solar Center, Thuwal 23955, Saudi Arabia

Dr. A. Savva

Cambridge University, Department of Chemical Engineering and Biotechnology, Philippa Fawcett Dr,

Cambridge CB3 OAS, United Kingdom

Dr. G. Wantz, Dr. Damien Thuau

Université de Bordeaux, CNRS Bordeaux INP/ENSCBP Laboratoire de l'Intégration du Matériau au Système

UMR 5218, 16 Avenue Pey Berland, 33607 Pessac Cedex, France

Dr. Lee J. Richter

Materials Measurement Laboratory

National Institute of Standards and Technology, Gaithersburg, Maryland 20899, USA

Dr. R. Nakar, Dr. O. Dautel

Institut Charles Gerhardt Montpellier, UMR 5253 CNRS-UM-ENSCM. ENSCM, 8 rue de l'Ecole

Normale, 34296 Montpellier Cedex 05, France

Dr. T. Nicolini, Prof. G. Hadziioannou, Prof. N. Stingelin

Université de Bordeaux, CNRS Bordeaux INP/ENSCBP, Laboratoire de Chimie des Polyméres Organiques

UMR 5629, Allée Geoffroy Saint-Hilaire, 33615 Pessac Cedex, France

Prof. N. Stingelin

School of Materials Science \& Engineering and School of Chemical \& Biomolecular Engineering, Georgia Institute of Technology, Atlanta, Georgia, USA

natalie.stingelin@gatech.edu

Keywords: mixed conduction, organic electrochemical transistors, hydrophilic conjugated polymers, poly(3-(6-hydroxy)hexyl thiophene)

Organic mixed conductors find use in batteries, bioelectronics technologies, neuromorphic computing and sensing. While great progress has been achieved, polymer-based mixed conductors frequently experience significant volumetric changes during ion uptake/rejection, i.e., during doping/de-doping, charging/discharging. Although ion dynamics may be enhanced in expanded networks, these 
volumetric changes can have undesirable consequences, e.g., negatively affecting hole/electron conduction and severely shortening device lifetime. Here, we present a new material poly[3-(6hydroxy)hexylthiophene] (P3HHT) able to transport ions and electrons/holes, as tested in electrochemical absorption spectroscopy and organic electro-chemical transistors, that exhibits low swelling, attributed to the hydroxylated alkyl side-chain functionalization. P3HHT displays a thickness change upon passive swelling of only $+2.5 \%$, compared to $+90 \%$ observed for the ubiquitous PEDOT:PSS, and $(+10$ to +15$) \%$ for polymers such as poly(2-(3,3'-bis(2-(2-(2methoxyethoxy)ethoxy)ethoxy)-[2,2'-bithiophen]-5-yl)thieno[3,2-b]thiophene) (p(g2T-TT)). Applying a bias pulse during swelling, this discrepancy becomes even more pronounced, with the thickness of P3HHT films changing by $<10 \%$ while the one of p(g2T-TT) structures increases by $(+75$ to +80$) \%$. Importantly, the initial P3HHT film thickness is essentially restored after de-doping while p(g2T-TT) remains substantially swollen. We, thus, expand the materials-design toolbox for the creation of lowswelling soft mixed conductors with tailored properties and applications in bioelectronics and beyond.

The demand for soft mixed conductors, e.g., for use in batteries [1] and organic bioelectronics device platforms [2] has led to numerous strategies for the rational chemical design of materials able to transduce ionic into electronic currents and vice versa[3]. One important feature that these materials must possess is the ability to take up ions. However, efficient ion uptake/flow is often accompanied by an intrinsic volumetric expansion of the active material, rendered more pronounced in case excess solvent is also incorporated into the material during ion uptake. Such an expansion/shrinkage can compromise mechanical integrity during cycling, limiting device stability as was shown for batteries[4] as well as for bioelectronics devices $[5,6]$ - and pronounced volumetric changes associated with ion insertion/intercalation have been recognized as one of the major limitations in lithium ion-based technologies[7].

Chemical design may assist in modulating the interplay between swelling-induced enhanced ion dynamics and undesirable effects on stability. In the bioelectronics area, targeted structures typically contain $\pi$ conjugated backbones to provide for good electronic charge transport; while flexible, often polar, side chains are used to assist with ion diffusion, to introduce solubility/processability and to aid with the materials' compatibility with biological environments. More specifically, one promising approach has relied on functionalizing conducting[8] and semiconducting[9, 10, 11] polymer backbones with 
hydrophilic substituents, such as glycolated side chains. This functionalization has proven to be extremely versatile, leading to materials that allowed successful fabrication of $p$-type[10], $n$-type and ambipolar[11] organic electrochemical transistors (OECT)s. However, oligo(ethylene glycol) (and similar) side chains render materials purification difficult; they also can lead to structural disorder such as backbone torsion both effects that can limit electron/hole transport [12]. Moreover, introduction of oligo(ethylene glycol) side chains frequently results in high sensitivity of important thermal phase transitions to environmental conditions (e.g., humidity). To give an example, the glass transition temperature, $T_{\mathrm{g}}$, of poly[3tetra(ethylene glycol)thiophene] — a material comprised of a polythiophene backbone and oligo(ethylene glycol) side chains - is notably affected by exposure to a humid environment[13]. In turn, the torsional backbone order and, hence, optoelectronic features significantly vary with temperature[13]. Structural[14] and mechanical properties also will be affected, rendering systematic establishment of structure/property relations intricate. In this communication, we demonstrate that in poly[3-(6-hydroxy)hexyl thiophene] (P3HHT; see inset in Figure 1a for chemical structure), hydroxylated alkyl side chains introduce mixed conduction when operated with aqueous electrolytes, as work on random copolymers of poly(3-hexyl thiophene) (P3HT) and P3HHT already indicated[15], but without excessive swelling and/or unwanted plasticizing effects observed in materials with oligoethylene-glycol side chains[13, 16, 17, 18]. The synthetic procedure and chemical characterization of the P3HHT are given in the Supplementary Information (Scheme S1, Figure S1-8).

In order to assess the swelling behavior of P3HHT upon exposure to aqueous electrolytes, we first characterized the volumetric change upon continuously flowing an aqueous solution of $0.1 \mathrm{~mol} / \mathrm{L} \mathrm{KCl}$ over P3HHT films, making a comparison to a reference sample in air (ambient relative humidity of (30 to 40) \%) using Quartz Crystal Microbalance with Dissipation monitoring (QCM-D). Initially, no electrical bias was applied to obtain insights into the "passive" swelling behavior of P3HHT (Figure 1b). We employed (92 \pm 4) $\mathrm{nm}$ thick films, and deduced changes in mass uptake from the measured frequencies, $f$, utilizing the Sauerbrey equation and converted these into thickness changes (see Supplementary Information for more 
details). $\mathrm{KCl}$ electrolyte solutions were used for these measurements as this allowed relatively straightforward comparison with literature data[19].

Reassuringly, based on the deduced mass uptake of the $\mathrm{P} 3 \mathrm{HHT}$ film $/ \mathrm{KCl}$ electrolyte systems, we infer a very minor thickness change $(+2.4 \%)$ of the polymer and, in turn, a nearly negligible volumetric change, assuming that the cross-sectional area is not affected by swelling in the configuration used for this material because we used films 'pinned' to a substrate (Figure 1c). This minute change is in contrast to the behavior of $\operatorname{poly}\left(2-\left(3,3^{\prime}-\right.\right.$ bis(2-(2-(2-methoxyethoxy)ethoxy)ethoxy)-[2,2'-bithiophen]-5-yl)thieno[3,2-b]thiophene) $(\mathrm{p}(\mathrm{g} 2 \mathrm{~T}-\mathrm{TT}))$, for which a volumetric (thickness) change of at least $(+10$ to +15$) \%$ was calculated under these conditions (see Figure 1b, QCM-D section of SI for multiple harmonics data, Figure S9, and modeling approach). The contrast is even stronger with respect to cross-linked poly(3,4ethylenedioxythiophene):polystyrene sulfonate (PEDOT:PSS), which swells by $\approx 85 \%$ in the same scenario[20]. Since P3HHT displays a similar surface hydrophilicity as, e.g., PEDOT:PSS, deduced from water contact-angle measurements (Figure S10), our initial "passive" swelling data suggests that P3HHT may feature a lower bulk hydrophilicity compared to PEDOT:PSS — and, likely, compared to p(g2T-TT) and other conjugated polymers with highly polar side-chain functionalization. While comparison with materials other than $\mathrm{p}(\mathrm{g} 2 \mathrm{~T}-\mathrm{TT})$ is rendered challenging as often different electrolytes and conditions were used in the literature, a passive swelling behavior as low as found for P3HHT was reported only for poly[6-(thiophen-3-yl)hexane-1-sulfonate tetramethylammonium]-co-poly(3-hexyl thiophene) (PTHS $\mathrm{TMA}^{+}$-co-P3HT) comprising a large fraction of $\mathrm{P} 3 \mathrm{HT}$ (77 mol\%; i.e., a relatively apolar material that does not allow significant OECT operation). PTHS TMA ${ }^{+}$-co-P3HT copolymers of low P3HT content featured similar behavior as p(g2T-TT)[21]; while poly(7-glycol-naphthalene-1,4,5,8-tetracarboxylic-diimide-bisthiophene)[22] (i.e., $\mathrm{p}(\mathrm{gNDI}-\mathrm{T} 2)$ ) seems to passively swell more than PEDOT:PSS (values extracted from literature are reported in Table $\mathbf{S 1}$ in the SI). 


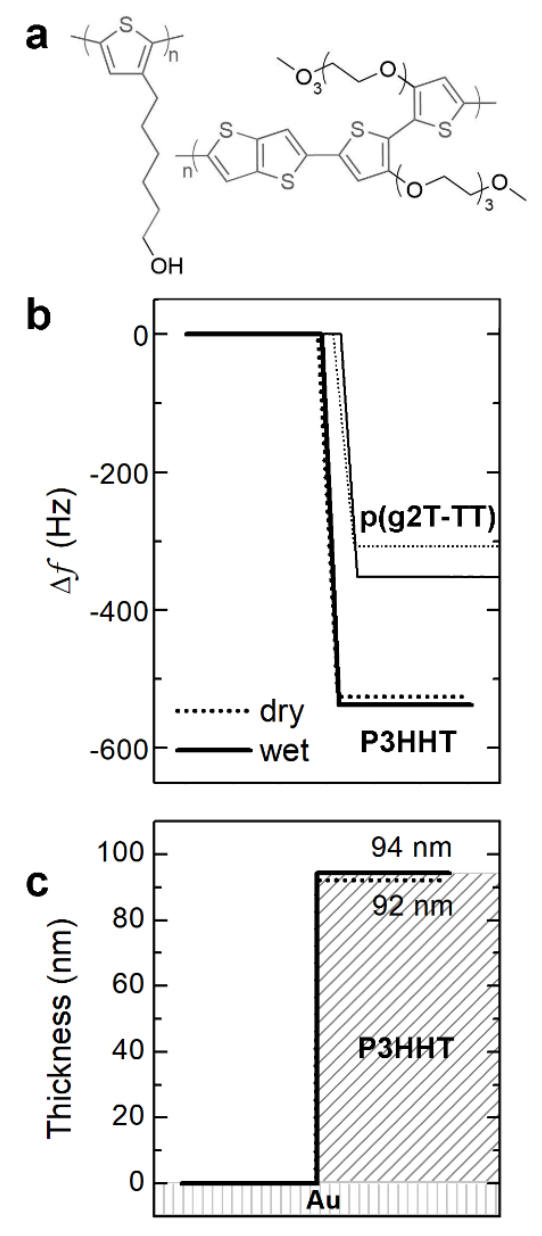

Figure 1 a) Chemical structures of P3HHT, left, and p(g2T-TT), right. b) $7^{\text {th }}$ Harmonic of QCM-D data obtained on P3HHT films with respect to a bare gold sensor reference. Passive swelling leads to negligible differences between the dry and wet state of a $90 \mathrm{~nm} P 3 H \mathrm{HT}$ film (bold lines) while QCM-D data for a $50 \mathrm{~nm}$ film of $\mathrm{p}(\mathrm{g} 2 \mathrm{~T}-\mathrm{TT})$ (thin lines) shows a more noticeable increase in frequency variation. c) Sauerbrey-derived thickness from the $7^{\text {th }}$ harmonic of the QCM-D for P3HHT in the wet and dry state (solid and dotted lines, respectively; further details are given in the SI).

We proceeded to investigate the electrolyte uptake behavior of both P3HHT and p(g2T-TT), using again QCMD but this time with a set-up equipped with an electrochemical module (e-QCM-D) to apply, in a pulsed fashion, a bias of $0.6 \mathrm{~V}$, which leads to ion/electrolyte injection into the bulk of the film structures (Figure 2). A few observations can be immediately made. We can infer from the small change in the oscillation frequency upon application of a voltage bias that P3HHT undergoes a small mass uptake upon electrolyte exposure/application of a bias pulse (Figure 2a,b). This is very different for $\mathrm{p}(\mathrm{g} 2 \mathrm{~T}-\mathrm{TT})$, where we observe a notable difference in frequency, $\Delta f$, in the presence/absence of an electric field, indicating a significant mass uptake upon application 
of the $0.6 \mathrm{~V}$ bias [Note that the frequency of the e-QCM-D oscillations changes when the mass on the sensor changes[23]; further details are given in the e-QCM-D section of the SI, in addition to frequency and dissipation data for multiple harmonics are given in Figure S11 and S12).]

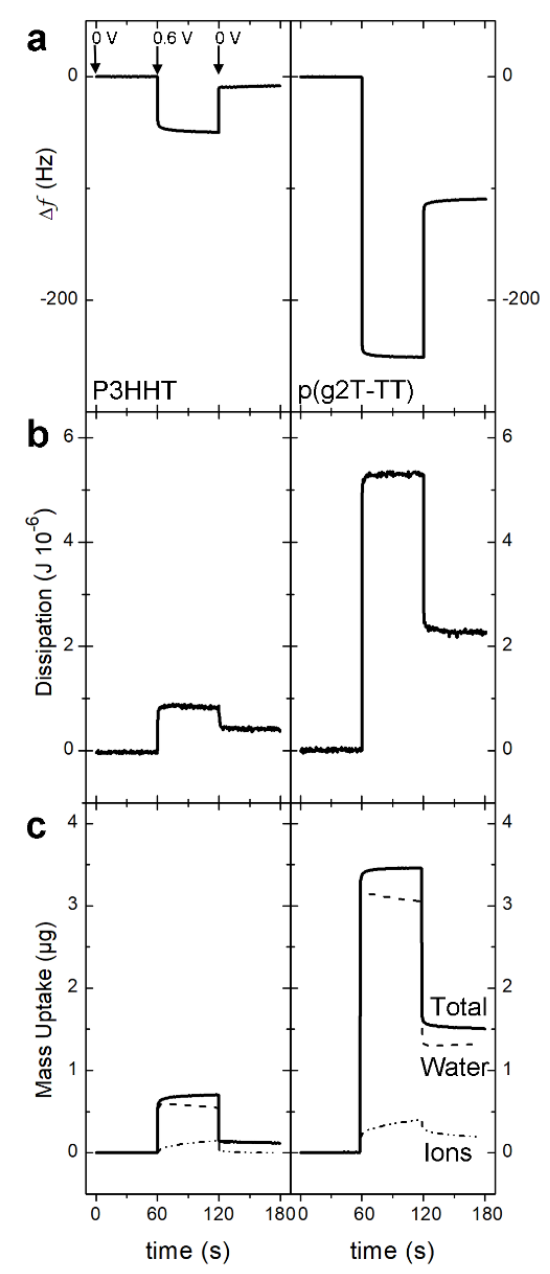

Figure 2 Comparison of e-QCM-D data for P3HHT and $p(g 2 T-T T)$ upon application of a bias pulse of $\Delta V=0.6 V$ showing minor changes in a) frequency and b) dissipation variation for P3HHT with respect to $p(g 2 T-T T)$. c) By fitting the frequency and dissipation data over multiple harmonics of the QCM, the mass uptake was calculated, from which a volumetric change can be deduced (see Supplementary Information for details). Notably different responses for the two polymers are found. Coupling eQCM-D results with electrical data recorded simultaneously, contributions to mass uptake from ions and water injected during application of a bias can be obtained, given here in dashed-dotted lines and dashed lines, respectively.

Additional information can be obtained from the dissipation of the signal - a measure of energy loss during sensor oscillation that reflects the softness of the sample[23]. We record relatively small changes in dissipation for P3HHT compared to p(g2T-TT), suggesting that swollen films of P3HHT are mechanically 
stiffer than $\mathrm{p}(\mathrm{g} 2 \mathrm{~T}-\mathrm{TT})$. This implies that P3HTT not only takes up less electrolyte (as deduced from the smaller change in frequency), but also appears less plasticized by the electrolyte solution.

Encouraged by our 'active-swelling' results, we went on and quantified the mass uptake in presence of an electrolyte solution and upon application of a $+0.6 \mathrm{~V}$ bias, using multiple harmonics of the QCM resonance frequency and dissipation, and taking into account the energy losses during vibrations, assuming the same density for both polymers. Remarkably, while we infer from the high mass uptake of $\mathrm{p}$ (g2T-TT) an expansion in thickness by $(+75$ to +80$) \%$ in this scenario, we calculate the thickness of P3HHT films to only increase by less than $+10 \%$ (around $+9 \%$; Figure 2c). Moreover, upon removal of the electrical bias, P3HHT almost fully restores its initial thickness as attested by the minor change in QCM-D frequency ( $\Delta f \approx-10 \mathrm{~Hz}$ ) between its initial state and after the bias is removed, which translates to a $+1.8 \%$ thickness increase. In contrast, $\mathrm{p}(\mathrm{g} 2 \mathrm{~T}-\mathrm{TT})$ remains substantially swollen, around ( +30 to +35$) \%$ of its initial thickness $(\Delta f \approx-110 \mathrm{~Hz})$. This difference seems to predominantly result from water retention after the electric field is switched "OFF". Indeed, separating the contribution of anions and water by combining e-QCM-D data with simultaneously recorded electrical data (see SI Figure S13 for details), we identify the remaining thickness change of P3HHT after bias removal to be due to the fact that $20 \%$ of overall water molecules are trapped in the films, but essentially no ions. In $\mathrm{p}(\mathrm{g} 2 \mathrm{~T}$ TT), $45 \%$ of the water molecules remain in the system as well as some ions (Figure 2c, right panel), explaining why, after the electrical field is switched "OFF", this material keeps its mechanical softness, typical for plasticized material, while P3HHT mostly recovers its materials stiffness, as already alluded to above (Figure 2b).

Intriguingly, the different swelling behavior between P3HHT and $\mathrm{p}(\mathrm{g} 2-\mathrm{TT})$ cannot be explained entirely by the significantly higher ion injection into $\mathrm{p}(\mathrm{g} 2 \mathrm{~T}-\mathrm{TT})$ at $0.6 \mathrm{~V}$. Comparison of the total mass uptake indicates that, for $\mathrm{P} 3 \mathrm{HHT}$, each injected $\mathrm{Cl}^{-}$is accompanied by $\approx 7$ water molecules, while for $\mathrm{p}(\mathrm{g} 2 \mathrm{~T}-\mathrm{TT})$, it is $\approx 15 \mathrm{H}_{2} \mathrm{O}$ molecules for each $\mathrm{Cl}^{-}$. At a lower bias of $0.2 \mathrm{~V}$, where we approximately achieve the same accumulated charge in both polymer films (see Figure S14 in SI), the ratio of $\mathrm{H}_{2} \mathrm{O}$ molecules for each $\mathrm{Cl}^{-}$increases for $\mathrm{p}(\mathrm{g} 2 \mathrm{~T}-\mathrm{TT})$ to $\approx 20$. This experimental evidence demonstrates that $\mathrm{p}(\mathrm{g} 2 \mathrm{~T}-\mathrm{TT})$ absorbs more water than P3HHT 
regardless of the bias that is applied, and it further highlights that $\mathrm{p}(\mathrm{g} 2 \mathrm{~T}-\mathrm{TT})$ consistently retains more solvent molecules after the bias is removed. This observation may explain the electrochemical stability of P3HHT structures when subjected to repeated cycling over increasing voltage ranges up to $0.75 \mathrm{~V}$ (see Figure S15). We hypothesize that this behavior is linked to the lower density of polar sites per repeat unit of P3HHT that can interact with water molecules, compared to those of $\mathrm{p}(\mathrm{g} 2 \mathrm{~T}-\mathrm{TT})$. While water retention in polymers depends on many factors, water-polymer interactions can be expected to be one of the main driving forces. The framework of a proposed hierarchy of interactions (cation-anion $>$ water-ion $>$ water-polar $=$ polar-polar $=$ water-water $>$ water-hydrophobic)[24], thereby, helps to understand the concert of competing forces acting in these complex systems and the propensity of a material to absorb and retain water molecules.

Important for its usage in devices such as OECTs, the ion uptake during application of a positive bias results in chemical doping of P3HHT. We used electrochemical absorption spectroscopy[25, 26], which is a commonly used method in the organic mixed conductor field[9,14-17] for the purpose of tracking ion uptake/doping (details on this methodology are given in the Supplementary Information). Data obtained using an $0.1 \mathrm{~mol} / \mathrm{L} \mathrm{KCl}$ solution as electrolyte and a similar measurement geometry as employed for the eQMC-D experiments, reveals bleaching of the ground-state absorption centered around $550 \mathrm{~nm}$ upon application of a $0.75 \mathrm{~V}$-bias while, simultaneously, an increase of the polaron band (centered around 800 $\mathrm{nm}$ ), characteristic for doped thiophene-based polymers, is observed (see Figure 3a). In agreement with our e-QMC-D data, upon removal of the bias, the same ground state absorption is restored (Figure 3b), indicating nearly complete de-doping, without the need to apply a negative bias.

This favorable behavior, in addition to the fact that the $\mathrm{OH}$-functionalization seems not to affect the hole mobility of materials as measured in a thin-film transistor (TFT) using P(3HT-3HHT) copolymers (see Supplementary Information Figure S16), has a direct impact on OECT device performance (Figure 3). For instance, the ease with which the material can be de-doped, as evidenced with our electrochemical spectroscopy measurements, leads to consistently low "OFF"-currents (see transfer characteristics displayed 
in Figure 3c). As a consequence, a high "ON"/"OFF" ratio of $10^{4}$, i.e., a pronounced difference between the "ON" and "OFF" current, is realized, which is on par/on the higher end of reported "ON"/"OFF" ratios
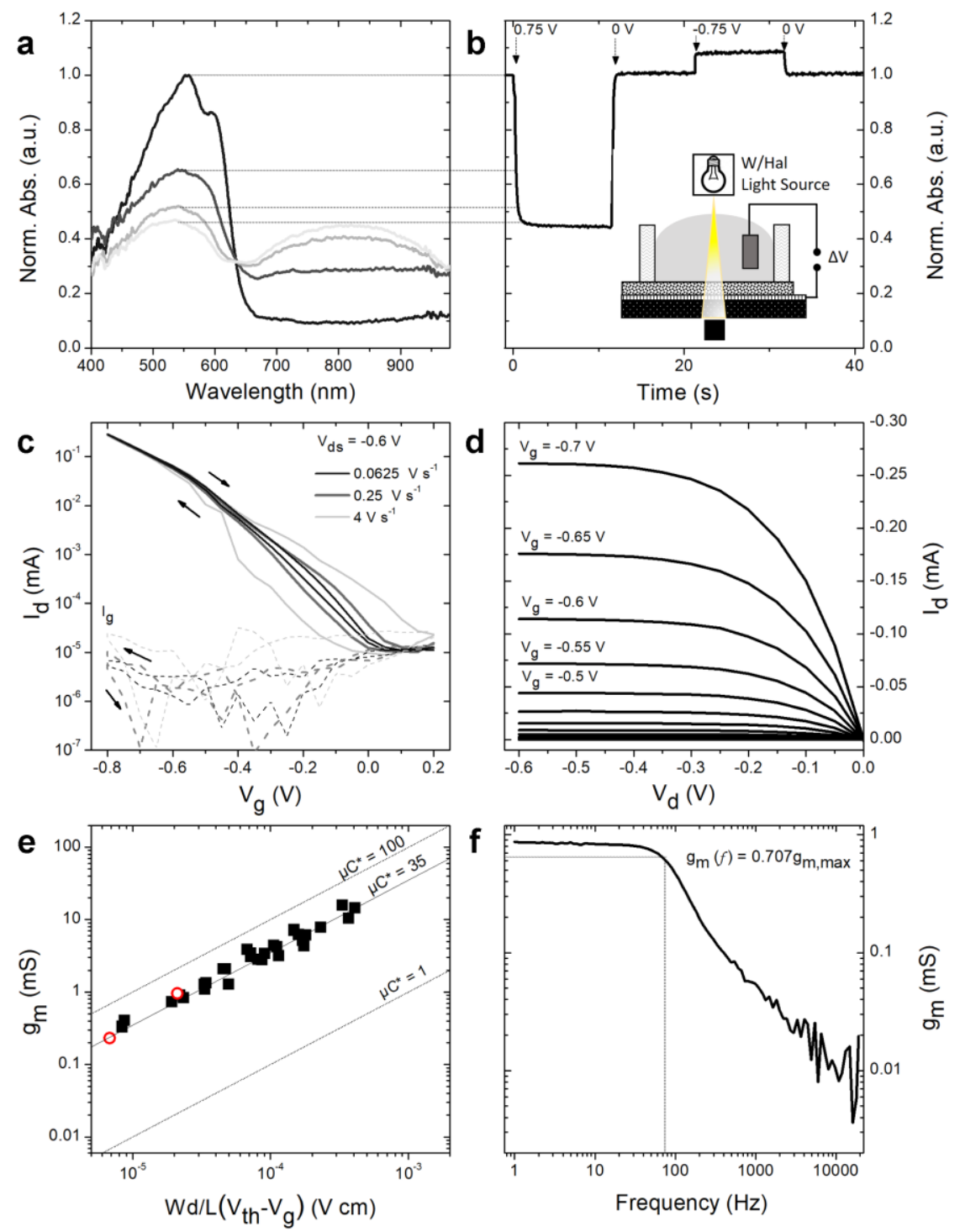

Figure 3 a) Time evolution of P3HHT absorption recorded upon application of $0.75 \mathrm{~V}$ bias. b) Absorption intensity at $\approx 560 \mathrm{~nm}$ normalized at $t=0 \mathrm{~s}$ versus time. c) Transfer loop characteristics of an unpatterned device $(\mathrm{W}=100 \mu \mathrm{m}, \mathrm{L}=10 \mu \mathrm{m})$ at varying sweep rates. d) Forward output characteristics of the same device using a $V_{d}$-scan rate of $0.5 \mathrm{~V} / \mathrm{s}$, while the gate voltage varied between (+0.1 to -0.7) V with voltage steps of $0.05 \mathrm{~V}$. e) Scaling of transconductance, $g_{m}$, at $V_{g}=0.6 \mathrm{~V}$ with channel dimensions using data obtained from simple, unpatterned devices (black squares) and patterned, insulated devices (red, empty circles; see details of channel dimensions and devices in SI). f) Bandwidth measurement of a patterned OECT $(\mathrm{W}=100 \mu \mathrm{m}, \mathrm{L}=10 \mu \mathrm{m})$ at $V_{d}$ $=-0.6$ V showing the half-power point to be at $75 \mathrm{~Hz}$. 
reported in literature on $p$-type devices $[9,10,21]$. Very clean output characteristics were also recorded (Figure 3d). [Note: In case ion flow in- and out- of the polymers is very slow when compared to the gate voltage sweeping times, and/or ions remain in the active OECT layer even when the gate voltage is removed, a higher "OFF"-current would be observed, similar to TFTs when the active layer is slightly doped[27] and free charges remain present when the device is switched "OFF". Gate leakage can contribute to a high “OFF"-current as well, but we generally observed low gate currents, $I_{\mathrm{g}}$, in the $1 \mathrm{nA}$-regime. This effect was further limited by using in some cases insulated, patterned devices (see OECT section of the SI for details on device fabrication)]

A large set of devices of different geometries (different channel lengths, $L$; channel widths, $W$; and thickness $d$; see Figure S17 in the SI for details), including two sets of insulated, patterned, small-channel devices with two different thicknesses, $d$ (see Figure S18), and simple, unpatterned devices, allowed us to extract the figure of merit often used to compare OECT device performance[28], that is $\mu \cdot C^{*}$, where $\mu$ is the OECT mobility and $C^{*}$ the volumetric capacitance, by plotting the transconductances, $g_{\mathrm{m}}=\frac{\partial I_{d}}{\partial V_{g}}$, deduced from the transfer characteristics, against $\frac{W \cdot d}{L \cdot\left(V_{t h}-V_{g}\right)}\left(V_{\text {th }}\right.$ is the threshold voltage, $V_{\mathrm{g}}$ is the gate voltage). We obtain $\mu \cdot C^{*}(\mathrm{OECT})$ to be $(35 \pm 2) \mathrm{F} \cdot \mathrm{cm}^{-1} \cdot \mathrm{V}^{-1} \cdot \mathrm{s}^{-1}$ (Figure 3e) which is only slightly lower to what is reported for PEDOT:PSS, $(47 \pm 2) \mathrm{F} \cdot \mathrm{cm}^{-1} \cdot \mathrm{V}^{-1} \cdot \mathrm{s}^{-1}$, but at the benefit of drastically lower volumetric expansion/retraction upon swelling.

The above discussion shows that P3HHT can be easily integrated in devices such as OECTs, leading to clean device characteristics that scale with device geometry (channel length, channel width; patterned $v s$. unpatterned) and come at par with devices fabricated, e.g., with the widely available PEDOT:PSS. Patterned devices also allowed us to perform accurate bandwidths measurements (Figure 3f) to obtain the cut-off frequency of such OECTs. This enables estimation of their time response. We extract a cut-off frequency of $75 \mathrm{~Hz}$. This is somewhat slower than what is measured for, e.g., p(g2T-TT). Yet, the lower swellability and, thus, the expected minimized structural changes to the film by water accumulation, could 
render $\mathrm{P} 3 \mathrm{HHT}$ and its derivatives attractive for sensing applications, e.g., for tracking of biological signals $[29,30]$. Indeed, preliminary OECT stability measurements suggest that P3HHT-based devices are only minimally affected by immersion in water over multiple days (see Figure S19), contrary to commonly processed PEDOT:PSS[5].

In conclusion, despite the plethora of possible chemical structures that can be designed and synthesized, so far, focus has been on the ubiquitous PEDOT:PSS and its derivatives, as well as common semiconducting polymers used in the organic thin-film transistor field, substituted with polar side chains such as oligoethylene-glycol moieties. We showed here that hydroxyl-alkyl functionalization provides an interesting, alternative side-chain option - especially if low swelling (i.e., small volumetric changes) upon doping is required. We attribute the low swelling behavior of P3HHT compared to glycolated organic mixed conductors to the fact that hydroxyl alkyl side-chains offers less sites available for water-polymer interactions, especially when compared to fully glycolated side-chain polymers such as $\mathrm{p}(\mathrm{g} 2 \mathrm{~T}-\mathrm{TT})$. The $\mathrm{OH}$-functionalization also has other benefits. It hardly affects the typical molecular packing; when compared to P3HT, only an increase in the lamellar stacking distance due to the slightly longer side-chain length is found (see structural characterization section in SI and GIWAXS data reported in Figure S20). As a consequence, electronic charge transport as assessed in TFTs is not negatively impacted by the OHfunctionalization. Use of hydroxyl-alkyl side chains also leads to a higher melting temperature, $\mathrm{T}_{\mathrm{m}},(\approx 290$ ${ }^{\circ} \mathrm{C}$; see Figure S21 for DSC thermogram) compared to, e.g., regio-regular P3HT of similar molar mass displays, $\mathrm{T}_{\mathrm{m}} \approx 240{ }^{\circ} \mathrm{C}$ to $260{ }^{\circ} \mathrm{C}[31,32,33]$, promising a somewhat higher temperature stability. The benefits come at an expense. In order to minimize the hysteresis between forward and backwards scans when measuring OECT transfer characteristics, relatively slow sweeping rates of $0.25 \mathrm{~V} \cdot \mathrm{s}^{-1}$ (compared to $\mathrm{p}(\mathrm{g} 2 \mathrm{~T}-\mathrm{TT})[10])$ are needed. In addition, the figure of merit of $\mu \cdot C^{*}$ (OECT) deduced for P3HHT, while rather comparable to PEDOT:PSS, is lower than for materials such as $\mathrm{p}(\mathrm{g} 2 \mathrm{~T}-\mathrm{TT})$ for which a $\mu \cdot C^{*}$ (OECT) of $(240 \pm 90) \mathrm{F} \cdot \mathrm{cm}^{-1} \cdot \mathrm{V}^{-1} \cdot \mathrm{s}^{-1}$ is reported[28]. Yet, the large error of the $\mu \cdot C^{*}(\mathrm{OECT})$ values reported for $\mathrm{p}(\mathrm{g} 2 \mathrm{~T}-\mathrm{TT})$ indicates potential issues with accurately determining this figure of merit for this 
material, possibly resulting from, among other things, its variations in glass transition temperature (due to, e.g., different plasticizing degrees, which will affect mass transport) and its generally strong dependence of properties on the environment. Clearly the use of hydroxyl-alkyl functionalization will expand future materials design options, especially when such side chains are combined with backbones with a better electronic charge-transport capability, such as the one used in $\mathrm{p}(\mathrm{g} 2 \mathrm{~T}-\mathrm{TT})[34]$. Considering that the synthetic pathway is relatively straight-forward, requiring only a protection/de-protection step and enabling synthesis of highly regioregular materials because the oxygen atom is well distanced from the aromatic moiety of the monomer and, thus, does not affect its reactivity during polymerization, our work promises an alternative chemical design tool towards more reliable mixed conducting structures. This is achieved via materials that undergo lower volumetric changes upon doping/de-doping cycles (as suggested by the high electrochemical stability of P3HHT structures when subjected to repeated cycling over increasing voltage ranges up to $0.75 \mathrm{~V}$ that we observe; see Figure S15), that display a smaller environmental sensitivity and feature higher thermal transition temperatures, while hole transport seems not to be affected, as thin-film transistor measurements show (Figure S16), likely because the OHfunctionalization does not affect molecular packing nor the edge-on orientation often found for thiophenebased polymers[35], as already alluded to above. We thus conclude that hydroxyl-alkyl functionalization leads to model systems that can assist to systematically establish relevant structure/property interrelations with respect to the fundamental requirements that organic materials need to fulfill to sustain ion flow/electrical conduction without adverse side effects such as excessive swelling.

\section{Acknowledgements}

The authors thank Jonathan Rivnay and Sahika Inal for highly fruitful discussion and preliminary OECT measurements. Tommaso Nicolini, Georges Hadziioannou and Natalie Stingelin are grateful for financial support provided by the IONBIKE RISE project, which has received funding from the European Union's Horizon 2020 research and innovation programme under the Marie Skłodowska-Curie grant agreement No 823989. Tommaso Nicolini, Georges Hadziioannou and Natalie Stingelin also acknowledge funding from 
the MARBLE project (IdEX). Olivier Dautel acknowledges funding from the MAPLE project (Institut Carnot Chimie Balard Cirimat). Lee J. Richter and Tommaso Nicolini thank NSLS-II for the access to the CMS BM11 beamline of the Brookhaven National Laboratory NY, USA which is a U.S. DOE Office of Science Facilities, at Brookhaven National Laboratory under Contract No. DE-SC0012704.

\section{Declaration of conflict of interest}

The authors declare no conflict of interest.

\section{References}

[1] S. Muench, A. Wild, C. Friebe, B. Häupler, T. Janoschka, U. S. Schubert, Chem. Rev. 2016, 116, 9438.

[2] G. G. Malliaras, Biochim. Biophys. Acta - Gen. Subj. 2013, 1830, 4286.

[3] J. Rivnay, R. M. Owens, G. G. Malliaras, Chem. Mater. 2014, 26, 679.

[4] Y. Liang, Y. Yao, Joule 2018, 2, 1690.

[5] S.-M. Kim, C.-H. Kim, Y. Kim, N. Kim, W.-J. Lee, E.-H. Lee, D. Kim, S. Park, K. Lee, J. Rivnay, M.H. Yoon, Nat. Commun. 2018, 9, 3858.

[6] M. Moser, T. C. Hidalgo, J. Surgailis, J. Gladisch, S. Ghosh, R. Sheelamanthula, Q. Thiburce, A. Giovannitti, A. Salleo, N. Gasparini, A. Wadsworth, I. Zozoulenko, M. Berggren, E. Stavrinidou, S. Inal, I. McCulloch, Adv. Mater. 2020, 32, 2002748.

[7] Y. Yuan, K. Amine, J. Lu, R. Shahbazian-Yassar, Nat. Commun. 2017, 8, 15806.

[8] S. Inal, J. Rivnay, A. I. Hofmann, I. Uguz, M. Mumtaz, D. Katsigiannopoulos, C. Brochon, E. Cloutet, G. Hadziioannou, G. G. Malliaras, J. Polym. Sci. Part B Polym. Phys. 2016, 54, 147.

[9] S. Inal, J. Rivnay, P. Leleux, M. Ferro, M. Ramuz, J. C. Brendel, M. M. Schmidt, M. Thelakkat, G. G. Malliaras, Adv. Mater. 2014, 26, 7450.

[10] A. Giovannitti, D.-T. Sbircea, S. Inal, C. B. Nielsen, E. Bandiello, D. A. Hanifi, M. Sessolo, G. G. Malliaras, I. McCulloch, J. Rivnay, Proc. Natl. Acad. Sci. 2016, 113, 12017.

[11] A. Giovannitti, C. B. Nielsen, D.-T. Sbircea, S. Inal, M. Donahue, M. R. Niazi, D. A. Hanifi, A. Amassian, G. G. Malliaras, J. Rivnay, I. McCulloch, Nat. Commun. 2016, 7, 13066.

[12] R. Noriega, J. Rivnay, K. Vandewal, F. P. V. Koch, N. Stingelin, P. Smith, M. F. Toney, A. Salleo, Nat. Mater. 2013, 12, 1038.

[13] M. J. Dyson, E. Lariou, J. Martin, R. Li, H. Erothu, G. Wantz, Paul D. Topham, Olivier Dautel, S. Hayes, P. N. Stavrinou, N. Stingelin Chem. Mater. 2019, 31, 6540.

[14] C. G. Bischak, L. Q. Flagg, K. Yan, T. Rehman, D. W. Davies, R. J. Quezada, J. W. Onorato, C. K. Luscombe, Y. Diao, C.-Z. Li, D. S. Ginger, J. Am. Chem. Soc. 2020, 142, 7434. 
[15] C. M. Pacheco-Moreno, M. Schreck, A. D. Scaccabarozzi, P. Bourgun, G. Wantz, M. M. Stevens, O. J. Dautel, N. Stingelin, Adv. Mater. 2017, 29, 1604446.

[16] A. Savva, C. Cendra, A. Giugni, B. Torre, J. Surgailis, D. Ohayon, A. Giovannitti, I. McCulloch, E. Di Fabrizio, A. Salleo, J. Rivnay, S. Inal, Chem. Mater. 2019, 31, 927.

[17] L. Q. Flagg, C. G. Bischak, J. W. Onorato, R. B. Rashid, C. K. Luscombe, D. S. Ginger, J. Am. Chem. Soc. 2019, 141, 4345.

[18] A. Savva, R. Hallani, C. Cendra, J. Surgailis, T. C. Hidalgo, S. Wustoni, R. Sheelamanthula, X. Chen, M. Kirkus, A. Giovannitti, A. Salleo, I. McCulloch, S. Inal, Adv. Funct. Mater. 2020, 30, 1907657.

[19] E. Stavrinidou, P. Leleux, H. Rajaona, D. Khodagholy, J. Rivnay, M. Lindau, S. Sanaur, G. G. Malliaras, Adv. Mater. 2013, 25, 4488.

[20] A. Savva, S. Wustoni, S. Inal, J. Mater. Chem. C 2018, 6, 12023.

[21] P. Schmode, D. Ohayon, P. M. Reichstein, A. Savva, S. Inal, M. Thelakkat, Chem. Mater. 2019, 31, 5286.

[22] A. Giovannitti, I. P. Maria, D. Hanifi, M. J. Donahue, D. Bryant, K. J. Barth, B. E. Makdah, A. Savva, D. Moia, M. Zetek, P. R. Barnes, O. G. Reid, S. Inal, G. Rumbles, G. G. Malliaras, J. Nelson, J. Rivnay, I. McCulloch, Chem. Mater. 2018, 30, 2945.

[23] D. Johannsmann, Phys. Chem. Chem. Phys. 2008, 10, 4516.

[24] S. P. Rowland, Water in Polymers, American Chemical Society, Washington, D. C., 1980. p. 4

[25] Y. Zhai, Z. Zhu, S. Zhou, C. Zhu, S. Dong, Nanoscale 2018, 10, 3089.

[26] K. Schwedtmann, S. Schulz, F. Hennersdorf, T. Strassner, E. Dmitrieva, J. J. Weigand, Angew. Chemie Int. Ed. 2015, 54, 11054.

[27] E. J. Meijer, C. Detcheverry, P. J. Baesjou, E. van Veenendaal, D. M. de Leeuw, T. M. Klapwijk, J. Appl. Phys. 2003, 93, 4831.

[28] S. Inal, G. G. Malliaras, J. Rivnay, Nat. Commun. 2017, 8, 1767.

[29] D. Khodagholy, T. Doublet, P. Quilichini, M. Gurfinkel, P. Leleux, A. Ghestem, E. Ismailova, T. Hervé, S. Sanaur, C. Bernard, G. G. Malliaras, Nat. Commun. 2013, 4, 1575.

[30] L. Wang, S. Xie, Z. Wang, F. Liu, Y. Yang, C. Tang, X. Wu, P. Liu, Y. Li, H. Saiyin, S. Zheng, X. Sun, F. Xu, H. Yu, H. Peng, Nat. Biomed. Eng. 2020, 4, 159.

[31] C. R. Snyder, R. C. Nieuwendaal, D. M. DeLongchamp, C. K. Luscombe, P. Sista, S. D. Boyd, Macromolecules 2014, 47, 3942.

[32] C. R. Snyder, E. D. Gomez, J. Polym. Sci. Part B Polym. Phys. 2016, 54, 1202.

[33] F. P. V. Koch, J. Rivnay, S. Foster, C. Müller, J. M. Downing, E. Buchaca-Domingo, P. Westacott, L. Yu, M. Yuan, M. Baklar, Z. Fei, C. Luscombe, M. A. McLachlan, M. Heeney, G. Rumbles, C. Silva, A. Salleo, J. Nelson, P. Smith, N. Stingelin, Prog. Polym. Sci. 2013, 38, 1978. 
[34] I. McCulloch, M. Heeney, M. L. Chabinyc, D. DeLongchamp, R. J. Kline, M. Cölle, W. Duffy, D. Fischer, D. Gundlach, B. Hamadani, R. Hamilton, L. Richter, A. Salleo, M. Shkunov, D. Sparrowe, S. Tierney, W. Zhang, Adv. Mater. 2009, 21, 1091.

[35] M. Pandey, N. Kumari, S. Nagamatsu, S. S. Pandey, J. Mater. Chem. C 2019, 7, 13323. 